\title{
The Age Structure of Populations of Saccharomyces cerevisioe*
}

\author{
JULIAN ADAMS \\ Division of Biological Sciences and Department of Human Genetics, \\ University of Michigan, Ann Arbor, Michigan 48109 \\ E. D. ROTHMAN \\ Departments of Statistics and Human Genetics, \\ University of Michigan, Ann Arbor, Michigan 48109 \\ AND \\ K. BERAN \\ Institute of Microbiology, Czechoslovak Academy of Science, Prague \\ Received 16 March 1980; revised 25 August 1980
}

\begin{abstract}
A discrete deterministic model is described for the growth of an age-structured population of yeast, Saccharomyces cerevisiae, incorporating recent information on the asymmetry of cell division and control of the cell cycle in this species. Solutions are obtained for the age structure of the population at equilibrium, and for the equilibrium distribution of relative frequency of cells through the cell cycle. The model is applied to experimental data on the changing age structure of nonequilibrium populations of yeast. The model predicts well both the transient behavior and the equilibrium structure of such populations. It is shown that the asymmetry of cell division explains (1) the excess of newly formed daughter cells in the population as compared to the frequency of older cells and (2) the damped oscillations in the frequencies of cells of different ages as demographic equilibrium is approached.
\end{abstract}

\section{INTRODUCTION}

Saccharomyces cerevisiae reproduces by budding. Abscission of the bud to form a daughter cell leaves a bud scar on the surface of the parent cell which can be observed with a fluorescent stain (Streiblová and Beran [23]) or under the scanning electron microscope. Daughter cells formed by abscission of a bud will be free of bud scars, whereas cells which have produced one bud and have therefore undergone one generation will have

*Supported in part by Department of Energy Contract EY-77-C-02-2828.

MATHEMATICAL BIOSCIENCES 53:249-263 (1981)

(C) Elsevier North Holland, Inc., 1981

52 Vanderbilt Ave., New York, NY 10017

$0025-5564 / 81 / 020249+15 \$ 02.50$ 
one bud scar. Buds are never formed at the site of an old scar, and so yeast cells may be aged by counting the number of bud scars on the cell surface. Cells have been observed that have undergone over 40 generations of division [19].

The ability to determine the age structure of populations of yeast cells by this technique, coupled with the detailed knowledge available on the physiology of the cell cycle of Saccharomyces cerevisiae, makes this organism ideal for experimental studies on demography.

Beran et al. $[3,4]$ have presented data on the change of the age structure in experimental populations of yeast and have shown that the numbers of cells of different ages undergo oscillations of decreasing magnitude until a demographic equilibrium is reached. A demographic model of such populations generated by Gani and Saunders [7] did not show such oscillations and predicted that approach to demographic equilibrium should be monotonically decreasing.

In this paper we develop a model of the proliferation of yeast populations which considers the distribution of cells within the cell cycle. We obtain results which describe the equilibrium demographic structure of yeast populations in terms of characteristics of the cell cycle. The model predicts that the frequencies of cells with different ages will exhibit damped oscillations as a demographic equilibrium is approached. We show that these oscillations are a consequence of the transit time through the cell cycle as suggested by [7] and the asymmetric nature of cell division in Saccharomyces cerevisiae. This model fits well data obtained from populations of $S$. cerevisiae which are approaching demographic equilibrium.

\section{THE MODEL}

It is now well known that cells of $S$. cerevisiae divide asymmetrically [1, 13]. When the mature bud abscisses from the parent cell to form the daughter cell, it is smaller in size than the parent cell, and consequently these cells have a longer cell cycle time than their parent cells. For convenience we will refer to daughter cells as being in age class 0 and to parent cells as being in age class 1 . Cells which have undergone more than one cycle of budding are labeled accordingly. There is also some evidence that cells which have undergone two cycles of budding (age class 2) have a shorter cell cycle time than cells which have undergone one [13]. However, such a difference, if it exists, is minor compared to the difference between cell cycle times of age class zero and age class one cells. Although the cell cycle times of cells of different ages may vary, it is generally believed that the time taken from bud initiation to abscission is constant [25]. In the following treatment we describe the model for the most general case where each age class has a unique cell cycle interval. However, for the most part 
results are presented for the case where all cells in age class one or above have the same cell cycle time. Figure 1 diagrams this model of the cell cycle of yeast.

A previous attempt [7] to model the dynamics of a yeast population considered the longer cycle time of age class 0 cells, but did not take into account the distribution of cells within the cell cycle. The model postulated instead simple probabilities governing the transition from one age class to the next. This is an oversimplification of the dynamics of such populations, as it assumes that cells which have just divided have the same probability of dividing again as cells which divided at earlier points in time.

It is now clear that the cell cycle of yeast not only has well-defined stages, but also has a series of unique and specific controls which regulate passage through the cycle $[11,12]$. Therefore any model of the dynamics of a yeast population should ideally take account of the distribution of cells within the cell cycle. In other cell types, previous approaches to modeling cell proliferation have divided the cell cycle into phases corresponding to well-defined biological stages, such as DNA synthesis $(S)$, premitotic phase
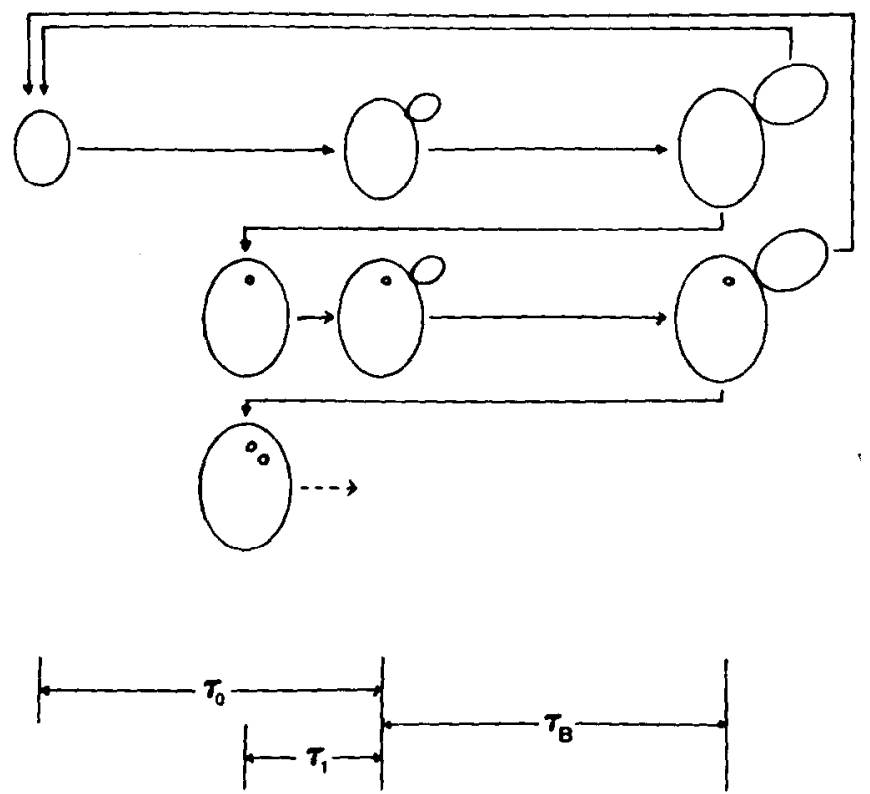

Fig. 1. Model of the cell cycle of Saccharomyces cerevisiae, showing relationship between generation number and number of bud scars. $\tau_{0}$, expected duration of unbudded phase of the cell cycle for age class 0 cells. $\tau_{1}$, expected duration of the unbudded phase of the life cycle for age class 1 cells. $\tau_{B}$, expected duration of budded phase. 
$\left(G_{2}\right)$, mitosis $(M)$, and the like. Passage through the cell cycle is specified by transition probabilities and also by maturity parameters [5, 17, 22, 24]. However, these approaches do not take into account the fact that biologically defined stages such as $S, M$, or $G_{2}$ almost surely represent a series of control events. In the model of the yeast population described below we have assumed that the cell cycle is divided into a number of subintervals, and thus the distribution of cells between the subintervals represents the distribution of position within the cell cycle. In this respect our model is analogous to the state vector model of Hahn [9] and the compartment models of Hopper and Klein and their coworkers [8, 14-16].

Each sector of the cell cycle, budded and unbudded, is considered to be divided into a number of subintervals of equal length. Although the number of subintervals is arbitrary in our model, they can be chosen to coincide with cell cycle control points such as have been described by Hartwell et al. $[11,12]$. Thus the model can be used to obtain information on the number of cells sensitive to a given control point [21].

Since the total number of cells in a yeast population is typically large $\left(\gg 10^{6}\right)$, a deterministic treatment for the proportions in each age class is sufficient. In the following model we designate

$$
\begin{aligned}
\nu_{t}^{0}(i, j)= & \text { the expected number of yeast cells with } i(0 \leqslant i<\infty) \text { bud scars } \\
& \text { in the } j \text { th }\left(1 \leqslant j \leqslant \kappa_{i}\right) \text { interval of the unbudded portion of the } \\
& \text { cell cycle, at time } t,
\end{aligned}
$$

and

$$
\begin{aligned}
\nu_{\ell}^{l}(i, j)= & \text { the expected number of yeast cells with } i(1 \leqslant i<\infty) \text { bud scars } \\
& \text { in the } j \text { th }\left(1 \leqslant j \leqslant \kappa_{B}\right) \text { interval of the budded portion of the cell } \\
& \text { cycle, at time } t .
\end{aligned}
$$

Similarly $\eta_{t}^{0}(i, j)$ and $\eta_{t}^{1}(i, j)$ represent the relative frequencies of these classes of yeast cells. The probability for age class $i$ of moving from one unbudded cell cycle subinterval to the next cell cycle subinterval during the time period $\Delta t$ is defined as $p_{i}$. Then $q_{i}=1-p_{i}$ is the probability of remaining in that subinterval. Thus the distribution of residence times in any subinterval is geometric. It follows from this that the cell cycle times should approximate a Pearson type III distribution. Bacterial cell cycle times fit such a distribution [20], and the data of Hartwell and Unger [13] suggest that it is a reasonable description of cell cycle times in yeast. Cell death is assumed to be small enough to be ignored. This assumption is justified by the results of Mortimer and Johnson [19], which showed no cell death for cells in age classes less than eight. For a constant time increment $\Delta t, p_{i}$ and $q_{i}$ will vary according to the age of the cell, since the time spent 
in the unbudded portion varies with cell age. If $\tau_{i}$ is the expected length of the unbudded portion of the life cycle for cells of age $i$, and this section of the life cycle is divided into $\kappa_{i}$ intervals, then

$$
p_{i}=\frac{\kappa_{i} \Delta t}{\tau_{i}}, \quad \text { with } \quad \kappa_{i} \Delta t<\tau_{i} .
$$

Similarly, $r$ is defined as the probability of moving from one budded cell cycle interval to the next cell cycle interval during the time period $\Delta t$, and $s=1-r$ is the probability of remaining in that interval. In the budded sector of the life cycle, $r$ and $s$ are constants whatever the age of the cell, as it is assumed that budding time is constant [25] and not dependent on age. If $\tau_{B}$ is the expected length of the budded portion of the life cycle and this section is divided into $\kappa_{B}$ intervals, then

$$
r=\frac{\kappa_{B} \Delta t}{\tau_{B}}, \quad \text { with } \quad \kappa_{B} \Delta t<\tau_{B} .
$$

The difference equations describing the change in the expected number of cells of different ages over time will be

$$
\begin{aligned}
\nu_{t+\Delta t}^{0}(0,1) & =q_{0} \nu_{t}^{0}(0,1)+r \sum_{i=0}^{\infty} \nu_{t}^{1}\left(i, \kappa_{B}\right), \\
\nu_{t+\Delta t}^{0}(i, j+1) & =p_{i} \nu_{t}^{0}(i, j)+q_{i} \nu_{t}^{0}(i, j+1), \quad 1 \leqslant j \leqslant \kappa_{i}, \\
\nu_{t+\Delta t}^{0}(i, 1) & =q_{i} \nu_{t}^{0}(i, 1)+r \nu_{t}^{1}\left(i-1, \kappa_{B}\right), \quad i \geqslant 1, \\
\nu_{t+\Delta t}^{1}(i, 1) & =p_{i} \nu_{t}^{0}\left(i, \kappa_{i}\right)+s \nu_{t}^{1}(i, 1), \quad i \geqslant 1, \\
\nu_{t+\Delta t}^{1}(i, j+1) & =r \nu_{t}^{1}(i, j)+s \nu_{t}^{1}(i, j+1), \quad 1 \leqslant j \leqslant \kappa_{B}-1 .
\end{aligned}
$$

Though the expected number of cells in any age class eventually increases exponentially, the proportion of cells in any one age class will approach an equilibrium. Total population size will increase according to the following equation:

$$
N_{t+\Delta t}=N_{t}\left[1+r \sum_{i=0}^{\infty} \eta_{t}^{\prime}\left(i, \kappa_{B}\right)\right],
$$

where $N_{t}$ is the total population size at time $t$. To obtain equilibrium solutions we first divide both sides of (3) by $N_{t+\Delta t}$ to obtain

$$
\eta_{e}^{0}(0,1)=\frac{r \lambda}{r \lambda+p_{0}},
$$


where $\eta_{e}^{0}(0,1)$ is the relative frequency at equilibrium of unbudded cells in age class 0 in the first interval of the cell cycle, and $r \lambda$ is the limiting form of the growth rate with $\lambda$ defined by

$$
\lambda=\sum_{i=0}^{\infty} \eta_{e}^{1}\left(i, \kappa_{B}\right) .
$$

It then follows from Eq. (3), (8), and (9) that the equilibrium frequencies of cells of age class 0 in the other subintervals of the unbudded phase of the cell cycle will be

$$
\eta_{e}(0, j)=\left[\frac{p_{0}}{p_{0}+r \lambda}\right]^{j-1}\left(\frac{r \lambda}{r \lambda+p_{0}}\right) .
$$

The remaining equilibrium frequencies of unbudded and budded cells in other age classes are given by the following recursion equations obtained from the appropriate difference equation (3)-(7) and Eq. (8):

$$
\begin{aligned}
& \eta_{e}^{1}(i, 1)=\frac{p_{i}}{r(\lambda+1)} \eta_{e}^{0}\left(i, \kappa_{t}\right), \quad i=0,1,2, \ldots, \\
& \eta_{e}^{0}(i, 1)=\frac{r}{r \lambda+p_{i}} \eta_{e}^{1}\left(i-1, \kappa_{B}\right), \quad i=1,2, \ldots, \\
& \eta_{e}^{1}(i, j)=\left(\frac{1}{\lambda+1}\right)^{j-1} \eta_{e}^{1}(i, 1), \quad i=1,2, \ldots, \quad j=1,2, \ldots, \kappa_{B}, \\
& \eta_{e}^{0}(i, j)=\left(\frac{p_{i}}{p_{i}+r \lambda}\right)^{j-1} \eta_{e}^{0}(i, 1), \quad i=0,1,2, \ldots, \quad j=1,2, \ldots, \kappa_{i} .
\end{aligned}
$$

It can be seen that the expected frequency of cells in each age class at equilibrium has a slowly decreasing geometric distribution as position in the cell cycle increases.

It may be of more interest from a practical standpoint to know, for each age class at equilibrium, the total proportions of cells in either the budded or unbudded phase of the life cycle. These results which may be obtained by summation over the appropriate subintervals, are presented in Table 1 for the special case where the expected cell cycle times of cells in age classes greater or equal to one, are the same (that is, where $p_{i}$ and $\kappa_{i}$ are constant for $i \geqslant 1$ ). The results in Table 1 consider that the cell cycle is divided into a discrete and arbitrary number of subintervals. In many cases the number of subintervals or indeed the subintervals themselves may be difficult to specify. In such situations a continuous time model of the cell cycle may be more appropriate. We can obtain results for the continuous time model by taking limits for the discrete model as $\Delta t \rightarrow 0$, in such a way that $\kappa_{0} \Delta t, \kappa_{1} \Delta t$, 
and $\kappa_{B} \Delta t$ approach finite limits which are less than $\tau_{0}, \tau_{1}$, and $\tau_{B}$ respectively. In particular, the equation for $\lambda$ in table 1 is replaced by

$$
e^{\rho \tau_{B}}=e^{-\rho \tau_{0}}+e^{-\rho \tau_{1}}
$$

where $\rho$ is the population specific growth rate [i.e. $\rho=(\ln 2) / T$, where $T$ is the population doubling time]. The continuous time results analogous to those in Table 1 are given in Table 2.

These results for the continuous time model can be shown to be equivalent to those of Hartwell and Unger [13], who used an alternative approach to obtain the expected frequencies of cell types at equilibrium.

\section{TABLE 1}

Discrete Time Equilibrium Solutions

$\begin{array}{lcc}\text { Age class } & \text { Unbudded } & \text { Relative frequency } \\ i=0 & \left(\frac{p_{0}}{p_{0}+r \lambda}\right)^{j-1} \frac{r \lambda}{r \lambda+p_{0}} & \frac{\lambda}{(\lambda+1)^{j}}\left[\frac{p_{0}}{p_{0}+r \lambda}\right]^{\kappa_{0}} \\ i>1 & \frac{r \lambda}{p_{1}}\left[\frac{p_{1}}{p_{1}+r \lambda}\right]^{j}(1-\theta) \theta^{i-1} & \frac{\lambda}{(\lambda+1)^{j}}\left[\frac{p_{1}}{p_{1}+r \lambda}\right]^{\kappa_{1}}(1-\theta) \theta^{j-1} \\ j=1,2, \ldots, \kappa_{1} & \kappa_{j=1,2, \ldots, \kappa_{B}}\end{array}$

${ }^{a}$ Relative frequency of cells in budded and unbudded phase of the cell cycle, where $\theta$ is defined by

$$
\theta=\left(\frac{p_{1}}{p_{1}+r \lambda}\right)^{\kappa_{1}}(1+\lambda)^{-\kappa_{B}}
$$

and $\lambda$ is the solution of the equation

$$
(1+\lambda)^{\kappa_{B}}=\left(\frac{p_{0}}{p_{0}+r \lambda}\right)^{\kappa_{0}}+\left(\frac{p_{1}}{p_{1}+r \lambda}\right)^{\kappa_{1}}
$$

TABLE 2

Continuous Time Equilibrium Solutions

\begin{tabular}{lccc} 
& \multicolumn{3}{c}{ Relative frequency $^{\mathrm{A}}$} \\
\cline { 2 - 4 } & Unbe class & Budded & Budded and unbudded \\
\hline$i=0$ & $1-e^{-\rho \tau_{0}}$ & $e^{-\rho \tau_{0}}\left(1-e^{-\rho \tau_{B}}\right)$ & $\phi$ \\
& $\left(1-e^{-\rho \tau_{1}}\right)(1-\phi) \phi^{i-1}$ & $e^{-\rho \tau_{1}}\left(1-e^{-\rho \tau_{s}}\right)(1-\phi) \phi^{i-1}$ & $(1-\phi)^{2} \phi^{i-1}$ \\
\hline
\end{tabular}

${ }^{2}$ Relative frequency of cells in the budded and unbudded phases of the life cycle, where $\phi=e^{-\rho\left(\tau_{1}+\tau_{B}\right)}$. 


\section{APPLICATION OF THE MODEL TO DATA OBTAINED ON THE GROWTH OF YEAST POPULATIONS}

\section{EXPERIMENTAL PROCEDURES}

The strain of Saccharomyces cerevisiae, media, and general growth conditions have been described previously [3]. Cells were grown aerobically in batch culture with glucose (Fig. 2, 3\% w/v; Fig. 3, 2.5\% w/v) as the carbon source. The culture volume was approximately $100 \mathrm{ml}$. Medium was added periodically to the cultures to ensure that the cells were in the exponential (unlimited) phase of growth throughout the duration of the experiments. Initial inocula consisted of more than $95 \%$ of unbudded cells in age class zero. These cells were prepared by centrifuging a population of cells on a dextran gradient as described previously [18] and isolating the densest fraction of cells in the gradient. Unbudded cells of age 0 are the smallest cells in the population [1]. There was no evidence of any physiological effect of the centrifugation process [18]. Data were collected on the growth rate of the population and the changing frequency of the various age classes. Cells were aged by counting the number of bud scars using the fluorescent stain procedure of Streiblova and Beran [23]. A detailed description of the method may be found in Beran [2]. This method is now standard for the estimation of the age of yeast cells (e.g. [2, 3, 4, 6, 10]). Since the sequence of budding follows a specific geographic pattern, occurring chiefly at the poles of the cell, and since the cells tend to roll on the microscope slide while the number of scars is being counted, all scars occurring on the surface of the cell can be counted. However, in practice it is difficult to count large numbers of bud scars accurately [6]. For this reason we grouped cells with more than 5 scars into one category. The frequency distributions of cells with different numbers of bud scars were determined from counts on 500-1000 cells in three independently prepared samples. Total cell number was determined hy counting in a hemocytometer.

\section{ESTIMATION OF PARAMETERS OF THE MODEL}

To apply the model to experimental data on the growth of yeast populations, estimates of the cell cycle times of the various age classes must be obtained. Data on the relative frequency of the age classes at demographic equilibrium can be used to estimate $\tau_{0}+\tau_{B}$ and $\tau_{1}+\tau_{B}$ provided that an independent estimate of the population specific growth rate $\rho$ be available. The estimate of $\rho$ from the data on total cell number can be obtained using standard least-squares regression procedures. In general this requirement does not present a serious limitation on the use of the estimation procedure. The distribution of the age classes in a sample from an infinitely large population is multinomial, and thus a convenient estimation proce- 
dure is maximum likelihood. Although theoretically the number of age classes is infinite, in practice it is difficult to assign cells with a large number of bud scars to a specific age class [6]. In our data we have therefore pooled all age classes above 5 . The likelihood function assuming a multinomial distribution of age classes at equilibrium is

$$
L(\phi)=C \phi^{n_{0}}\left[(1-\phi) \phi^{h}\right]^{M} \prod_{i=1}^{h}\left[(1-\phi)^{2} \phi^{i-1}\right]^{n_{i}},
$$

where $\phi$ is the expected frequency of cells in age class 0 (see Table 2), $n_{i}$ is the number of cells observed in age class $i, M$ is the total number of cells for all age classes greater than $h$, and $C$ is a constant representing the combinatorial term.

The maximum likelihood estimator for $\phi$ can be obtained by straightforward techniques and is

$$
\hat{\phi}=\frac{n_{0}+\sum_{i=1}^{h}(i-1) n_{i}+h M}{n_{0}+\sum_{i=1}^{h}(i+1) n_{i}+(h+1) M} .
$$

Although this estimator is biased, the magnitude of the bias is dependent on the magnitude of $\phi^{h}$. Since $\phi$ is usually between 0.5 and 0.6 , with $h$ equal to 5 or 6 the bias is small. This is reasonable, as the expected numbers of cells in the age classes higher than 5 are vanishingly small. The parameters $\tau_{0}+\tau_{B}$ and $\tau_{1}+\tau_{B}$ are monotonic functions of $\phi$, and so the maximum likelihood estimates of these parameters are found in the obvious manner. If data are available on the frequency of unbudded and budded cells in each age class, then the parameters $\tau_{0}, \tau_{1}$, and $\tau_{B}$ may be estimated separately.

\section{FITTING OF THE MODEL TO THE DATA}

The model described here predicts two important features concerning the growth of yeast populations:

(1) Whenever the generation time of cells in the zero age class is longer than those of higher age classes, zero age class cells will constitute more than $50 \%$ of the population at equilibrium (see Table 2). There is now abundant evidence in the literature to substantiate this prediction (e.g. [13]). The simpler model of Gani and Saunders [7] also predicts that zero class cells will be present in frequencies larger than $50 \%$ if their generation time is larger. However, in fitting their model to data of Beran et al. [3], it is difficult to understand why they set the zero age class frequency to a value 
less than $50 \%$. Though this frequency may drop below $50 \%$ due to sampling variation, it can do so in the population only if zero age class cells have a shorter expected generation time than others. This possibility is not considered in their paper or indeed by anyone.

(2) The second and more interesting prediction of the model is that the frequency of the different age classes will undergo damped oscillations until demographic equilibrium is reached. We will show that these damped oscillations are a consequence of the longer expected generation time of zero age class cells.

Oscillations in the frequency of different age classes have been reported before [2-4]. To test the ability of our model to describe such oscillations the model was fitted to the data gathered by one of us (K.B.) on the changing age structure of yeast populations. Population cell cycle times $\left(\tau_{0}+\tau_{B}\right.$ and $\left.\tau_{1}+\tau_{B}\right)$ were estimated using Eq. (18) from the age distributions at the last time points. Thus we assume that at these points demographic equilibrium had been reached. The predicted changes in the age structure of the populations were calculated from these estimates and Eqs. (3)-(7). The numbers of subintervals were determined from Eqs. (1) and (2) with a time increment $\Delta t$ chosen to be 5 minutes. Choice of a time increment smaller than this value had no detectable effect on the predicted changes. The distribution of the cells of age 0 within the cell cycle in the initial inocula was assumed to be geometric or triangular and such that in the initial inoculum, cells at the beginning of the cell cycle were most frequent, and those at the end of the cycle least frequent. This distribution of cells in the initial inocula is expected for two reasons: (1) There is a correlation of position in the cell cycle with size [1], and therefore the fraction of cells isolated from the density-gradient centrifugation would be mostly buds recently abscissed from the parent cells, as these are the smallest cells in the population. The probability of finding a cell more advanced in the cell cycle would decrease with increasing size of the cell. (2) The density gradient centrifugation procedure is not completely efficient in isolating small cells. Nevertheless the relative frequency of larger cells in the densest fraction decreases the larger the cell. In our simulations there was no significant difference between the results obtained assuming an initial discrete triangular and a geometric distribution. Figures 2 and 3 show the results of two experiments with different glucose concentrations and the change in relative frequency of the cells of different ages predicted from the model. Not only is the period of the oscillations predicted well, but also their magnitude. The estimate of specific growth rate for the population in Fig. 3 was faster than that for Fig. 2, and consequently the estimates of daughter cell cycle time $\left(\tau_{0}+\tau_{B}\right)$ and parent cell cycle time $\left(\tau_{1}+\tau_{B}\right)$ were not greatly different. The asymmetry of cell division in Saccharomyces increases as the generation 


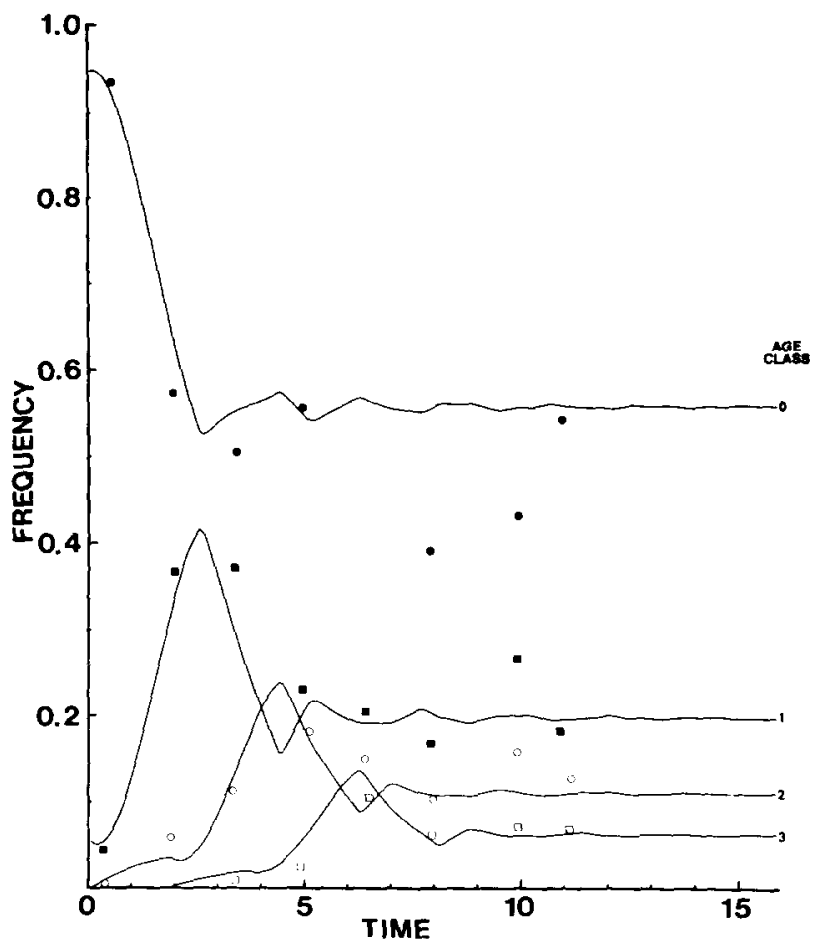

Fig. 2. Observed changes of cells of different ages over time (hours). Solid lines represent predicted changes. Observed changes are shown by the symbols $\bullet$, age class 0 ; $\square$, age class $1 ; 0$, age class $2 ; \square$, age class 3 . Estimates used in calculating the predicted changes were $\hat{\rho}=0.320 \mathrm{hr}^{-1} ;\left(\tau_{0} \hat{+} \tau_{B}\right)=2.55 \mathrm{hr} ;\left(\tau_{1} \hat{+} \tau_{B}\right)=1.83 \mathrm{hr}$. The maximum likelihood estimate of the frequency of cells in age class 0 is $\phi=0.56$.

time of the population increases [11]. It can be seen for data in Fig. 3, where cell division is almost symmetric, that the amplitudes of the oscillations are much smaller than those in Fig. 2. These results suggest that the damped oscillations in the frequencies of the various age classes are a consequence of the asymmetry of cell division. Direct proof of this requires the analysis of a set of integrodifferential equations which have been refractory to solution. We have therefore resorted to numerical evaluation of Eqs. (3)-(7). Figure 4 shows the results for one set of values of daughter and parent cell cycle times. The values for $\tau_{0}, \tau_{1}$, and $\tau_{B}$ were obtained from the empirical relationships described by Hartwell and Unger [13] between $\tau_{0}, \tau_{1}, \tau_{B}$, and the population-specific growth rate. Consequently these values are biologically reasonable. It can be seen that when parent and daughter cell cycle 


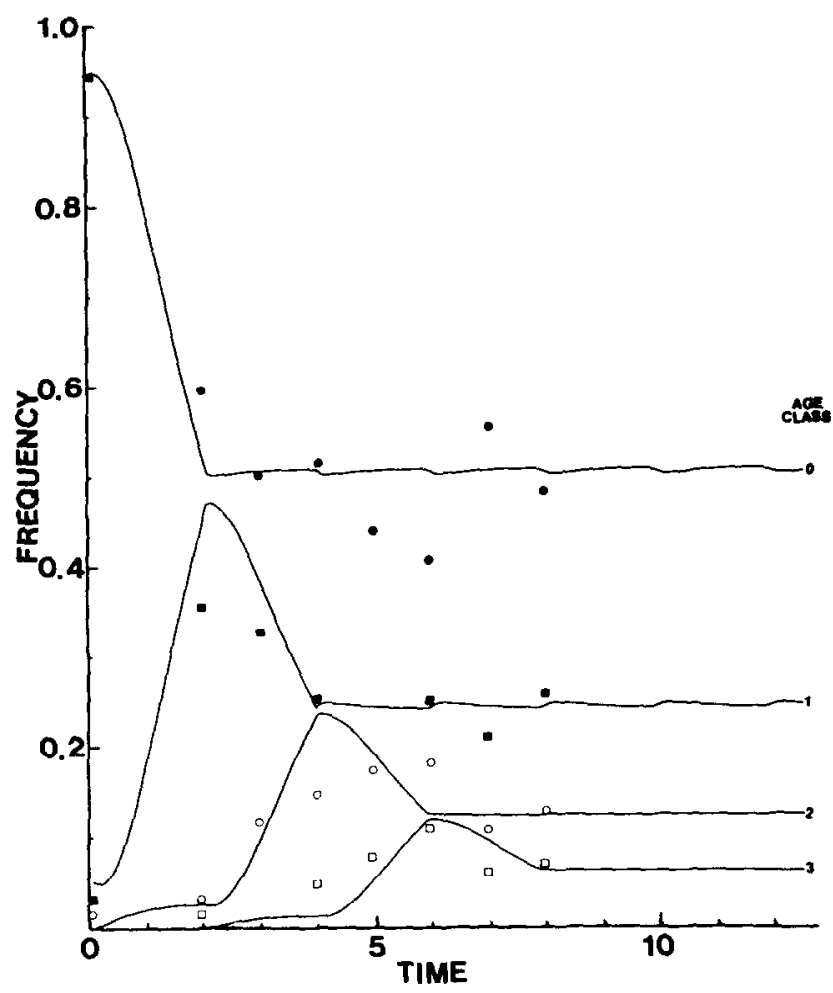

Fig. 3. Observed changes of cells of different ages over time (hours). Solid lines represent predicted changes. Observed changes are shown by the symbols $\bullet$, age class 0 ; $\square$, age class $1 ; 0$, age class $2 ; \square$, age class 3 . Estimates used in calculating the predicted changes were $\hat{\rho}=0.350 \mathrm{hr}^{-1} ;\left(\tau_{0} \hat{+} \tau_{B}\right)=2.02 \mathrm{hr} ;\left(\tau_{1} \hat{+} \tau_{B}\right)=1.95 \mathrm{hr}$. The maximum likelihood estimate of the frequency of cells in age class 0 is $\phi=0.51$.

times are equal, equilibrium is approached monotonically. Whenever the daughter cell cycle time is greater than that of the parents, damped oscillations in the frequencies of the age classes appear, and the magnitude of the oscillations is directly proportional to the degree of asymmetry of cell division. Thus the damped oscillations seen in Fig. 2 are a consequence of the asymmetry of cell division in this population.

\section{CONCLUSIONS}

The discrete, deterministic model presented here describes well the frequencies of the different age classes in a population of Saccharomyces cerevisiae at equilibrium. For the simple case where budded and unbudded cells are considered together and where all cells in age class 1 and above 


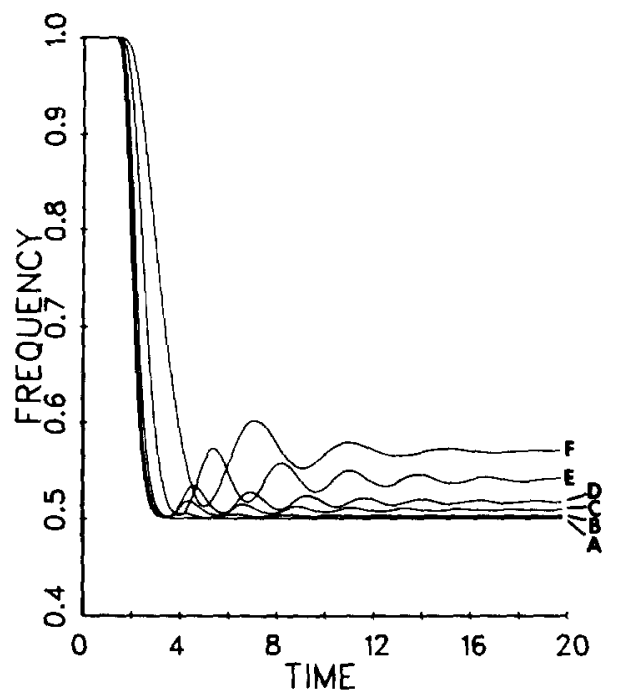

Fig. 4. Expected change in the frequency of age class 0 cells over time (hours), showing the oscillatory approach to demographic equilibrium with asymmetric cell division. A: $\tau_{0}+\tau_{B}=2.17 \mathrm{hr} ; \tau_{1}+\tau_{B}=2.17 \mathrm{hr}$. B: $\tau_{0}+\tau_{B}=2.20 \mathrm{hr} ; \tau_{1}+\tau_{B}=2.17 \mathrm{hr}$. C: $\tau_{0}+\tau_{B}=2.33 \mathrm{hr} ; \tau_{1}+\tau_{B}=2.22 \mathrm{hr}$. D: $\tau_{0}+\tau_{B}=2.52 \mathrm{hr}$; $\tau_{1}+\tau_{B}=2.28 \mathrm{hr}$. E: $\tau_{0}+\tau_{B}=3.18$ hr; $\tau_{1}+\tau_{B}=2.53 \mathrm{hr}$. F: $\tau_{0}+\tau_{B}=4.62 \mathrm{hr} ; \tau_{1}+\tau_{B}=3.08 \mathrm{hr}$.

behave identically, the model makes the same predictions as those of Gani and Saunders [7] and Hartwell and Unger [13]. Under the assumption that all cells have the same cell cycle time, the relative frequencies of the different age classes can be described simply by $\left(\frac{1}{2}\right)^{n+1}$, where $n$ is the number of bud scars. However, cells with no bud scars have significantly longer cell cycle times than other cells, and under these circumstances zero age class cells will occur in the population at equilibrium with a frequency of greater than $50 \%$. This percentage increases as the differences between generation times of zero class cells and other cells increases (see Tables 1 and 2). The equilibrium frequency of cells within the cell cycle is described by a slowly decreasing geometric distribution.

Under nonequilibrium conditions our results show that the frequencies of cells of different ages in nonequilibrium populations undergo damped oscillations until demographic equilibrium is reached. These oscillations are a function of the longer cell cycle times of zero age class cells. The model presented here predicts well the oscillations observed in experimental batch cultures of Saccharomyces cerevisiae, even though this model is still a simplification of the actual biological phenomena involved. We have assumed for the sake of computational ease that all cells in age class 1 and 
above have the same cell cycle times. There is some evidence that this is not true and that cell cycle times tend to decrease as the cell grows older. Nevertheless, the correspondence between the predicted and observed results suggests that any difference in cell cycle times for cells in age class one or above is minor.

\section{REFERENCES}

1 J. Adams, The interrelationship of all growth and division in haploid and diploid cells of Saccharomyces cerevisiae, Exptl. Cell Res. 106:267-275 (1977).

2 K. Beran, Budding of yeast cells; their scars and aging, Adv. Microbial Physiology 2:143-171 (1968).

3 K. Beran, E. Streiblova and J. Lieblová, On the concept of the population of the yeast Saccharomyces cerevisiae, in Proceedings of the 2nd Symposium on Yeasts (A. KockováKratochivilová et al,, Eds.), 1969, pp. 353-363 Publ. House Slovak Acad. Sci., Bratislava.

4 K. Beran, I. Malek, E. Streiblová, and J. Lieblova, The distribution of relative age of cells in yeast populations, in Microbial Physiology and Continuous Culture (E. O. Powell, C. G. T. Evans, R. E. Strange, and D. W. Tempest, Eds.), H.M.S.O., 1967.

5 F. J. Burns and I. F. Tannock, On the existence of a $G_{0}$ phase in the cell cycle, Cell Tissue Kinet. 3:321-334 (1970).

6 B. L. A. Carter and M. N. Jagadish, The relationship between cell size and cell division in the yeast Saccharomyces cerevisiae, Exptl. Cell Res. 112:15-24 (1978).

7 J. Gani and I. W. Saunders, Fitting a model to the growth of yeast colonies, Biometrics 33:113-120 (1977).

8 M. Guiguet, B. Klein, and A.-J. Valleron, Diurnal variation and the analysis of percent labelled mitosis curves, in Biomathematics and Cell Kinetics (A.-J. Valleron and P. D. M. MacDonald, Eds.), 1978, pp. 191-198. Elsevier/North-Holland, Amsterdam.

9 G. M. Hahn, State vector description of the proliferation of mammalian cells in tissue culture. I. Exponential Growth, Biophys. J. 6:275-290 (1966).

10 M. Hayashibe, Cytodifferentiation in the cell cycle of yeasts, in Growth and Differentiation in Microorganisms (I. Ishikawa, Y. Maruyanla, and H. Matsumiya, Eds.), 1975, pp. 165-191. Japan Scientific Societies Press.

11 L. H. Hartwell, Saccharomyces cerevisiae cell cycle, Bacteriol. Rev. 38:164-198 (1974).

12 L. H. Hartwell, J. Culotti, J. R. Pringle, and B. J. Reid, Genetic control of the cell division cycle in yeast, Science 183:46-51 (1974).

13 L. H. Hartwell and M. W. Unger, Unequal division in Saccharomyces cerevisiae and its implications for the control of cell division, J. Cell. Biol. 75:422-435 (1977).

14 J. Hopper, A stochastic model applicable to both normal and stimulated cell populations, in Biomathematics and Cell Kinetics (A.-J. Valleron and P. D. M. MacDonald, Eds.), 1978, pp. 83-88. Elsevier/North-Holland, Amsterdam.

15 J. Hopper and P. Brockwell, Analysis of data from cell populations with circadian rhythm, in Biomathematics and Cell Kinetics (A.-J. Valleron and P. D. M. MacDonald, Eds.), 1978, pp. 211-221. Elsevier/North-Holland, Amsterdam.

16 B. Klein and $M$. Guiguet, Relative importance of the phases of the cell cycle for explaining diurnal rhythms in cell proliferation in tissues with a long $G_{1}$ duration, in 
Biomathematics and Cell Kinetics (A.-J. Valleron and P. D. M. MacDonald, Eds.), 1978, pp. 199-210. Elsevier/North-Holland, Amsterdam.

17 J. L. Lebowitz and S. I. Rubinow, Grain count distributions in labelled cell populations, J. Theoret. Rinl. 23:99-123 (1969).

18 J. Lieblová, K. Beran, and E. Streiblová, Fractionation of a population of Saccharomyces cerevisiae by centrifugation in a dextran gradient, Folia Microbiol. 9:205213 (1964).

19 R. K. Mortimer R. K. and J. R. Johnston, Life span of individual yeast cells, Nature 183:1751-1752 (1959).

20 E. O. Powell, Some features of the generation times of individual bacteria, Biometrika 42:16-44 (1955).

21 E. D. Rothman and J. Adams, Cell cycle control in Saccharomyces cerevisiae. Time of action of execution points, in preparation.

22 J. A. Smith and L. Martin, Do cells cycle? Proc. Nat. Acad. Sci. U.S. A. 70:1263-1267 (1973).

23 E. Streiblova and K. Beran, Demonstration of yeast scars by fluorescence microscopy, Exp. Cell Res. 30:603-605 (1963).

24 S. Svetina, An extended transition probability model of the variability of cell generation times, Cell Tissue Kinet. 10:575-581 (1977).

25 H. K. von Meyenburg, Energetics of the budding cycle of Saccharomyces cerevisiae during glucose limited aerobic growth, Arch. Mikrobiol. 66:289 303 (1969). 